\title{
Indicateurs de qualité dans le domaine stationnaire
}

\author{
Maria Wertlia, Sima Nuschin Djalalib, Omar Kherad ${ }^{c}$, Markus Schneemannº, Silvana K. Rampinib, \\ Adrian Rohrbasserd, Joël Lehmann ${ }^{e}$, Brigitte Zirbs Savigny ${ }^{\text {, }}$ Marc Michael Jungid, Mirjam Rodella Sapiad, \\ Jacques Donzéc, Regula Capaulf \\ a Prof. Dr méd. présidente de la commission qualité de la Société Suisse de Médecine Interne Générale SSMIG; b PD Dr méd., membre de la commission \\ qualité de la Société Suisse de Médecine Interne Générale SSMIG; ' Prof. Dr méd., membre de la commission qualité de la Société Suisse de Médecine \\ Interne Générale SSMIG; ${ }^{d}$ Dr phil., membre de la commission qualité de la Société Suisse de Médecine Interne Générale SSMIG; ${ }^{e}$ M.A., membre de la \\ commission qualité de la Société Suisse de Médecine Interne Générale SSMIG; ${ }^{\dagger}$ Dr méd., co-présidente de la Société Suisse de Médecine Interne Générale \\ SSMIG, membre de la commission qualité de la Société Suisse de Médecine Interne Générale SSMIG
}

La commission qualité de la Société Suisse de Médecine Interne Générale publie pour la première fois une liste d'indicateurs pouvant être utiles dans le domaine stationnaire. Il est important d'utiliser des indicateurs pour renforcer des mesures d'amélioration de la qualité dans le cadre d'un processus structuré.

L'une des principales préoccupations de la médecine interne générale (MIG) est la qualité de la prise en charge et du traitement des patientes et patients qui présentent de plus en plus des pathologies multiples (multimorbidité). Depuis de nombreuses années, la Société Suisse de Médecine Interne Générale (SSMIG) s'engage pour l'amélioration de la qualité et de l'efficacité du système de santé suisse [1]. Les obligations visant à mesurer la qualité et à la rendre visible ont en effet augmenté [2]. La commission qualité de la SSMIG s'est donc penchée de manière approfondie sur le

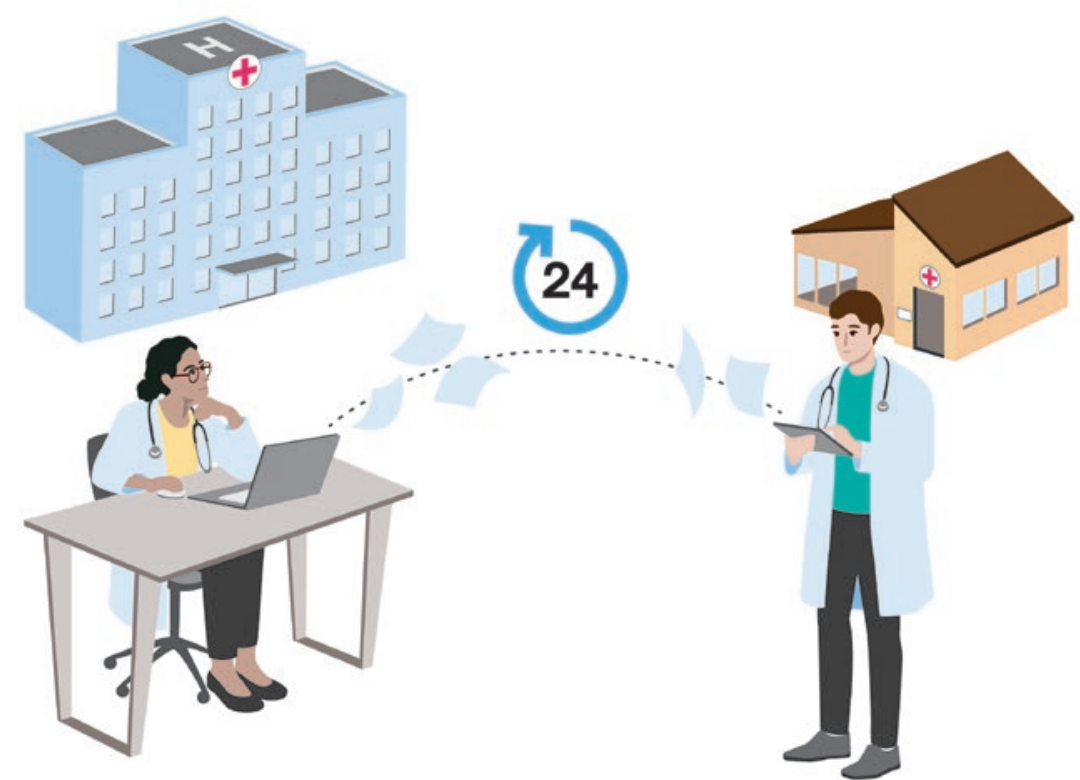

Le flux d'information est un indicateur pour la qualité de la prise en charge centrée sur la patiente ou le patient. thème de la mesure de la qualité et publie pour la première fois une liste d'indicateurs pouvant être utilisés dans le domaine stationnaire. Il est important d'utiliser des indicateurs pour renforcer des mesures d'amélioration de la qualité dans le cadre d'un processus structuré [3]. La qualité médicale est souvent décrite selon trois dimensions [4]: qualité structurelle, qualité des processus et qualité des résultats.

La SSMIG émet six recommandations pour la médecine stationnaire. Quatre indicateurs s'intéressent à la qualité des soins centrés sur les besoins du patient, un indicateur reflète la qualité générale des soins et un autre indicateur se réfère à la santé des collaboratrices et collaborateurs.

\section{Qualité de la prise en charge centrée} sur la patiente ou le patient

1. Proportion de rapports de sortie provisoires transmis dans les 24 heures au médecin chargé du suivi ambulatoire. Il doit contenir des informations sur les diagnostics, les procédures et les médicaments (avec les raisons éventuelles des changements de médication). Cet indicateur a pour objectif de transmettre rapidement les principales informations au médecin chargé du suivi afin de garantir la coordination du traitement lors de la transition. Une information efficace et rapide vise à améliorer la continuité des soins et à réduire le nombre de réhospitalisations, ainsi que de complications évitables.

2. Proportion de personnes âgées ( $\geq 65$ ans) chez lesquelles une nouvelle benzodiazépine a été intro- 
duite durant l'hospitalisation. Cet indicateur a pour objectif de réduire les nouvelles prescriptions de benzodiazépines au cours d'une hospitalisation afin d'éviter les effets secondaires inhérents à ces traitements.

3. Proportion de personnes âgées ( $\geq 65$ ans) à qui l'on a demandé si elles ont chuté au cours des 12 derniers mois, et le cas échéant, le nombre de chutes et le déroulement de la chute. Cet indicateur a pour but d'identifier les patients présentant un risque accru de chute, chez lesquels une intervention préventive s'avère judicieuse.

4. Proportion de patientes et patients recevant une transfusion lorsque le taux d'hémoglobine $(\mathrm{Hb})$ est supérieur à $8 \mathrm{~g} / \mathrm{dl}$. Cet indicateur a pour objectif de réduire le nombre de transfusions potentiellement non indiquées.

\section{Qualité générale des soins}

5. Proportion de rapports d'incidents (Critical Incidence Reporting System [CIRS]) pour des personnes hospitalisées en médecine interne générale (MIG) analysés et discutés par l'équipe soignante. L'objectif de l'indicateur est de stimuler une culture active de l'erreur en analysant et discutant les cas de CIRS.

\section{Santé des collaboratrices et collaborateurs}

6. Proportion de collaboratrices et collaborateurs en

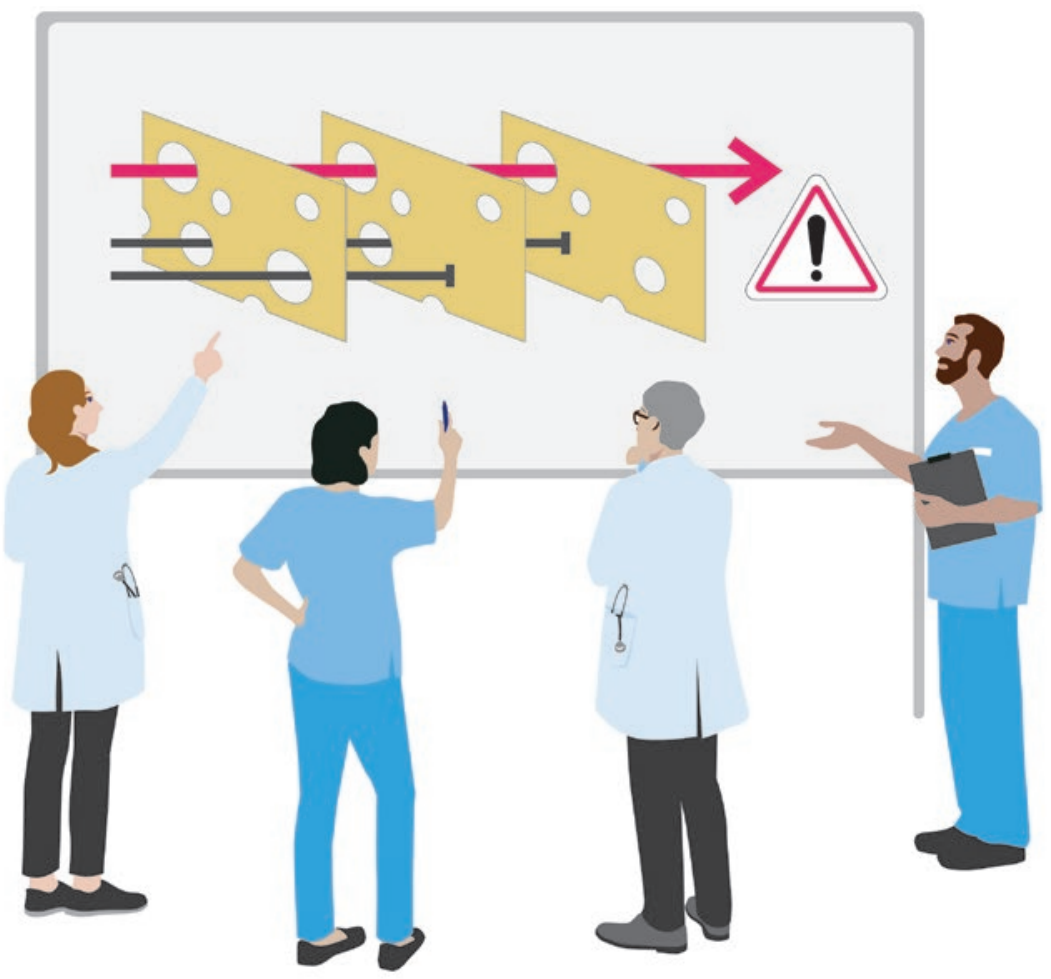

Critical Incidence Reporting System: les cas seront analysés et discutés. contact potentiel avec du sang ou des matériaux contaminés par le sang et présentant une protection vaccinale suffisante contre l'hépatite $B$. Cet indicateur a pour objectif de prévenir la transmission de l'hépatite B et d'améliorer la sécurité sur le lieu de travail.

\section{Indicateurs de qualité non recommandés}

La SSMIG s'exprime également sur trois indicateurs qu'elle ne peut recommander. Ces indicateurs ne sont pas adaptés à la médecine interne générale stationnaire, notamment en raison du caractère imprécis de leur mesure et de l'absence de preuves de leur efficacité. Il est important de reconnaître que des indicateurs imprécis peuvent donner lieu à des interprétations erronées, et donc se révéler préjudiciables.

Ces dernières années, le besoin et les exigences en matière de mesure de la qualité et de la visibilité de celle-ci ont gagné en importance.

1. Proportion de réhospitalisations évitables. L'indicateur a pour objectif d'identifier les hospitalisations potentiellement évitables et d'obtenir une amélioration de la qualité en analysant les causes (suivant l'exemple de l'indicateur utilisé par l'association nationale pour le développement de la qualité dans les hôpitaux et les cliniques (ANQ)). La principale critique concernant cet indicateur est que la méthode de mesure ne discrimine pas avec suffisamment de précision les cas de réhospitalisation qui auraient réellement pu être évités. En outre, il manque des preuves cliniques solides montrant que l'utilisation de cet indicateur entraîne une amélioration de la qualité des prises en charge. Comme l'attribution ne se fait pas selon des critères transparents et que l'algorithme (évitable/inévitable) est difficile à comprendre, il ne peut pas être utilisé en clinique, ni être influencé par les médecins.

2. Satisfaction des patientes et patients par rapport à leur séjour dans un hôpital pour des soins aigus (suivant l'exemple de la saisie par l'association ANQ). Cet indicateur a pour objectif de représenter une satisfaction générale à l'égard d'une hospitalisation comme substitut à la qualité de traitement. La satisfaction des patientes et patients ne peut être assimilée à la qualité du traitement et ne constitue donc pas un indicateur approprié pour améliorer la qualité du traitement. En outre, la définition de la «satisfaction générale» manque de clarté pour permettre d'en déduire des mesures efficaces à mettre en place. A l'inverse, la mesure de l'expérience des 


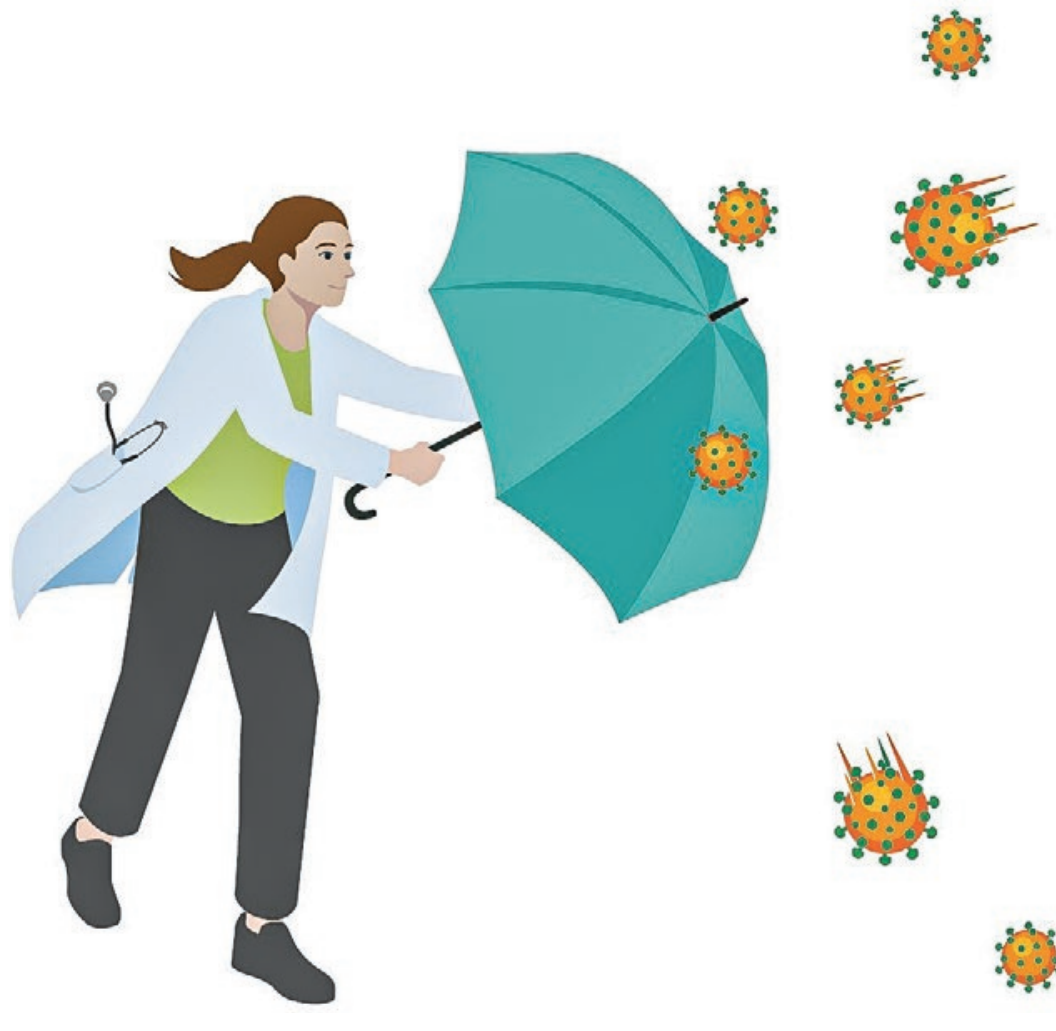

Prévenir la transmission de I’hépatite B, améliorer la sécurité au travail.

patientes et patients (patient experience) serait plus appropriée pour collecter des indications concernant les améliorations à apporter. La méthode de mesure de l'enquête ANQ ne comprend pas l'ensemble des patientes et patients et l'ajustement en fonction de facteurs confondants importants n'est pas suffisant pour permettre la comparaison entre les différents hôpitaux (benchmarking irrecevable).

3. Indicateurs de résultats recueillant la survenue de complications thérapeutiques évitables dans un établissement médical, sous la forme d'un indicateur de performance. Exemples: les mesures utilisées par l'association ANQ concernant la prévalence des escarres (nombre de patientes et patients hospitalisés souffrant d'escarres nosocomiales à un temps donné) et la prévalence des chutes (nombre de patientes et patients hospitalisés à un temps donné, ayant chuté au cours de l'hospitalisation en cours). Les taux des complications indésirables comme substitut de la qualité du traitement doivent, le cas échéant, être mesurés sous la forme d'enquêtes exhaustives. Un benchmarking plus approfondi ne devrait être effectué qu'après ajustement pour des variables cliniques confon- dantes récoltées de manière suffisamment détaillée. Les indicateurs de résultat devraient en outre être complétés par des indicateurs de processus pertinents qui reflètent aussi les efforts déployés pour influencer favorablement le résultat. Une institution présentant une prévalence faible d'escarre et de chutes en milieu hospitalier qui ne recourt à aucune mesure de prévention doit être évaluée différemment d'une institution à prévalence plus élevée, mais appliquant systématiquement des mesures de prévention.

Une description détaillée des indicateurs, la littérature spécialisée et de plus amples informations sont disponibles sur le site de la SSMIG (www.sgaim.ch/qualitaet). Dans un second temps, la commission qualité de la SSMIG élaborera des supports concernant les indicateurs qui renforcent les processus d'amélioration de la qualité. Cette documentation sera mise à disposition des membres de la SSMIG.

\section{Processus de création de la liste}

Une analyse de la littérature a permis d'identifier au total 36 indicateurs de processus potentiellement appropriés, 15 indicateurs de résultats et quatre indicateurs de qualité structurelle. La qualité structurelle inclut p. ex. le nombre de soignants par malade ou la maintenance adéquate des appareils techniques. La qualité des processus comprend p. ex. le traitement d'une maladie selon l'état actuel des connaissances. La qualité des résultats mesure p. ex. le taux de guérison, les réhospitalisations évitables, la satisfaction des patientes et patients ou la mortalité.

En février 2020, la commission qualité a analysé les in-

\section{Il est important de reconnaître que des indica-} teurs imprécis peuvent mener à des interprétations erronées et porter préjudice.

dicateurs dans le cadre d'ateliers et jugé pertinents six indicateurs stationnaires et six indicateurs ambulatoires. Pour la commission, il était important que les indicateurs puissent être utilisés au quotidien, afin de renforcer des mesures d'amélioration de la qualité en médecine interne générale. Ont été ainsi identifiés en premier lieu des indicateurs de processus. La commission a décidé de ne pas utiliser d'indicateurs de résultats, car leur saisie est très complexe et n'est généralement pertinente qu'en combinaison avec des indicateurs de processus. Les indicateurs sélectionnés ont été formulés, et leur applicabilité ainsi que leur mesurabilité ont été optimisées dans le cadre d'un processus 
itératif. Tous les indicateurs ont été vérifiés selon les critères également appliqués par l'American College of Physicians (ACP) [5]:

1. Importance: l'utilisation de l'indicateur entraînera une amélioration mesurable et significative des critères cliniques (impact important, écart de performance).

2. Traitement approprié: éviter la sur- ou sous-utilisation de mesures médicales.

3. Preuve clinique de haute qualité: des études ont révélé que l'utilisation de l'indicateur permet également d'améliorer le traitement.

4. Validité et fiabilité: l'indicateur permet de détecter l'événement à mesurer et fournit des résultats fiables en cas de mesures répétées.

5. Applicabilité: les événements à mesurer peuvent être influencés par les médecins, la mesure est réalisable de façon pratique (clarté, charge de travail).

Plusieurs spécialistes médicaux (médecins-cheffes et -chefs MIG, expertes et experts en économie de la santé) ont ensuite procédé à un examen externe.

\section{Défi relatif à la mise en œuvre}

L'objectif de la commission qualité était d'identifier, sur la base de la littérature spécialisée et de l'importance clinique, des indicateurs susceptibles de soutenir les processus d'amélioration de la qualité en MIG. La condition préalable essentielle est toutefois de créer des méthodes de mesure fiables. Pour que de nombreux indicateurs puissent être récoltés, les systèmes d'information électronique hospitaliers doivent être adaptés et les résultats collectés de manière systématique et uniforme. Il est par ailleurs impératif que les ressources humaines et financières nécessaires à la mesure de la qualité soient pleinement prises en compte.

Les indicateurs détaillés de qualité dans le domaine stationnaire sont en ligne sous le lien suivant: https://www.sgaim.ch/fr/qualité/qualite-a-lhopital.html
Références

1 Bull Med Suisses. 2021;102(17):572-3.

2 Le rapport national sur la qualité indique un grand potentiel d'amélioration du système de santé suisse, OFSP 8.11.2019.

3 Recommandations de l'Académie Suisse des Sciences Médicales, Bull Med Suisses, 2009;90(26-27):1044-54.

4 Avedis Donabedian. Evaluating the Quality of Medical Care. The Milbank Quarterly. 2005;83(4):691-729.

5 MacLean $\mathrm{CH}$. Time Out - Charting a Path for Improving Performance Measurement. N Engl J Med. 2018;378:1757-61.

Veuillez aussi lire l'interview avec Madame la Prof. Dr méd. et phil. Maria Wertli, présidente de la commission qualité de la Société Suisse de Médecine Interne Générale, sur le site du Bulletin des médecins suisses: "Indicateurs de qualité - Avantages et limites»

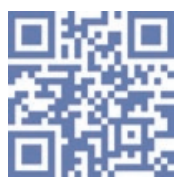

\section{Remerciements}

Nous remercions tous les collègues et experts pour leur examen critique et leurs suggestions: Dr Philippe Luchsinger (président de mfe), Prof. Drahomir Aujesky (représentant de la MIG des hôpitaux universitaires), PD Dr Thomas Brack et PD Dr Robert Escher (président et secrétaire de I'Association des médecins-chefs et -cadres internalistes suisses ([AMCIS]), Prof. Luca Gabutti (Association Smarter Medicine) et Prof. Alexander Geissler (School of Management, School of Medicine, HSG, Saint-Gall) et Dr méd. Lars Clarfeld (Secrétaire Général de la SSMIG). Nous remercions également Ursula Käser (Responsable du Service de la qualité, formation continue et postgraduée) pour son grand travail et son soutien.

\section{L'essentiel en bref}

- La SSMIG a formulé six indicateurs pour un traitement hospitalier de haute qualité de patients de plus en plus multimorbides.

- Les indicateurs de qualité concernent la qualité de la prise en charge centrée sur le patient, la qualité générale des soins et la santé du personnel. En outre, la SSMIG décrit trois indicateurs qu'elle ne recommande pas, à savoir la proportion de réhospitalisations évitables, la satisfaction des patients et la mesure des indicateurs de résultats sous la forme d'une enquête par sondage.

- Les indicateurs sélectionnés ont été examinés selon les critères suivants: importance, traitement approprié, preuves cliniques de haute qualité, validité et fiabilité, et applicabilité. 